\title{
Sistema de Alerta Agrícola en el Valle de SAN LorEnZO, TAMBOGRANDE
}

\section{Agricultural Alert System in the Valley of SAN Lorenzo, TAMBogrande}

\author{
Ninell Dedios ${ }^{1}$ y Mario Montero ${ }^{2}$ \\ ${ }^{1}$ Servicio Nacional de Meteorología e Hidrología - SenamHi, Urbanización Miraflores, Piura \\ ${ }^{2}$ Universidad Nacional de Piura, Campus Universitario s/n, Urbanización Miraflores, Castilla
}

\section{RESUMEN}

\begin{abstract}
Con el objetivo de dotar a quienes trabajan la tierra de información agrometeorológica actualizada, que permita minimizar o evitar las pérdidas causadas por agentes meteorológicos extremos, como sequías e inundaciones o eventos relacionados al cambio climático, incluidos los ataques nocivos (plagas o enfermedades) a los cultivos, el Servicio Nacional de Meteorología e Hidrología del Perú, SENAMHI, implementó un sistema para la vigilancia agrometeorológica. En este sentido, se desarrollaron talleres de formación y capacitación a los agricultores para que puedan conocer y utilizar la información generada para tomar decisiones adecuadas.
\end{abstract}

Descriptores: alerta agrícola

\begin{abstract}
With the aim of providing updated agrometeorologic information to land workers, which would minimize or avoid losses caused by meteorological extreme agents such as droughts and floods or climate change related events, including also the harmful assaults of plagues or diseases to crops, the National Service of Meteorology and Hydrology of Peru (SENAMHI) implemented a system for agrometeorologic alert. In this respect SENAMI developed workshops for the development and training of farmers with the goal that they learn to use the information generated to make suitable decisions.
\end{abstract}

Keywords: agricultural alert

\section{INTRODUCCIÓN}

La Agrometeorología es una disciplina que vincula los elementos o parámetros meteorológicos en su manifestación, periodicidad y variabilidad con los requerimientos de las especies cultivadas y su aprovechamiento económico.

En este sentido, los parámetros o elementos meteorológicos que pueden ser medidos u observados en una estación son muy variados.
Pueden ser utilizados con diferentes fines, como para las actividades agrícolas y el mejor manejo agronómico o para prevenir los impactos producidos por fenómenos naturales, como los efectos del fenómeno El Niño, que provocan la reducción de oferta de productos en el mercado local, nacional, internacional [Jiménez, 2000]. Asimismo, el cambio climático y sus efectos, provocados tanto por causas naturales [1] como antrópicas [2], ocasionan variabilidad climática a nivel local, regional y global con incidencia en la economía. 
Por ello, la información meteorológica representa una herramienta indispensable para ser usada por empresarios y productores en la gestión y organización eficiente de las labores de campo, desde la fecha de siembra, pasando por la frecuencia de riego y la fertilización, entre otros. No obstante, la problemática se centra en la falta de integración entre el productor (agricultores y empresarios) y los resultados generados desde las investigaciones científicas, así como en el vacío de información climática en el valle de San Lorenzo.

De esta manera, el objetivo del Sistema de Alerta Temprana en el valle de San Lorenzo es lograr una formación para anticiparse y actuar a tiempo.

\section{MÉTODO EXPERIMENTAL}

\section{Materiales y métodos}

El ámbito de desarrollo del proyecto se circunscribe al valle de San Lorenzo, que cuenta con un área bajo riego de 41550 ha cultivadas de mango, limón, arroz y algodón, con un sistema de riego regulado y no regulado (fig.1).

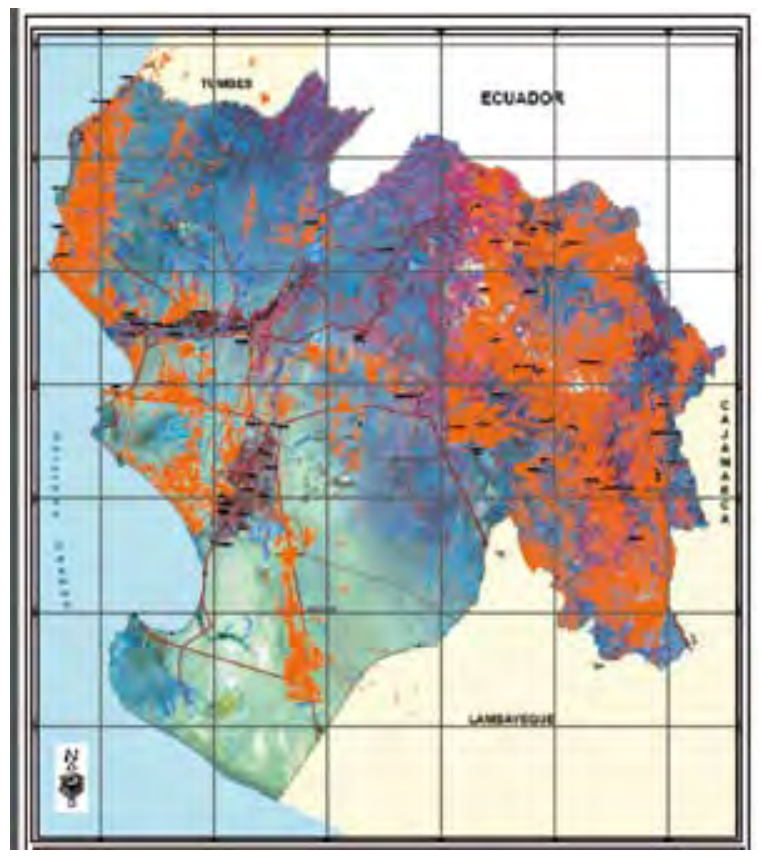

Figura 1: Área de estudio.

\section{Implementación de la estación agrometeorológica}

Seinstalóunaestación automática agrometeorológica Vantage Pro 2 Plus Inalámbrica Agrícola (D162W1) (figs. 2 y 3), en el sector Pedregal, valle de San Lorenzo, Tambogrande, Perú. Se consideró dicha ubicación de acuerdo a las normas internacionales de la OMM y se eligieron parcelas de observación fenológica de los cultivos representativos en el valle (mango y limón).

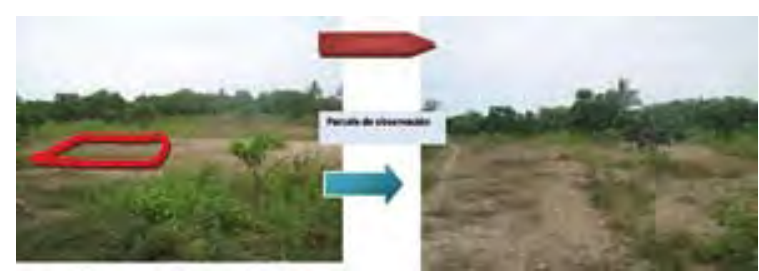

Figura 2: Estación automática Figura 3. Parcela fenológica.

\section{Integración en el sistema de gestión agrometeorológica y actores locales}

La divulgación de la información agrometeorológica regional se realiza desde los boletines informativos que publica mensualmente el Servicio Nacional de Meteorología e Hidrología (SENAMHI), los cuales constituyen parte del material de apoyo formativo a los agricultores del valle de San Lorenzo (fig. 4).

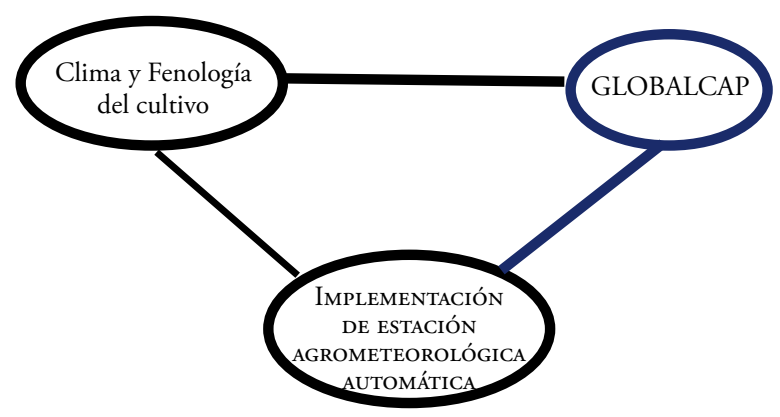

Figura 4: Esquema metodológico desarrollado para la toma de decisiones.

Fuente: Propia.

De otro lado, la metodología de difusión y aprendizaje considera la estructura de trabajo en serie: ver, juzgar y actuar, aplicada en los diferentes talleres del proyecto [3]. De manera general, se emplearon dos metodologías en la implementación del proyecto: La formación y capacitación a nivel de agricultores, y la implementación de la estación agrometeorológica, antes mencionada.

\section{Formación y capacitación a nivel de agricultores}

Consideramos a la educación como un elemento indispensable para alcanzar el desarrollo sostenible. La difusión de la realidad ambiental y agrícola en 


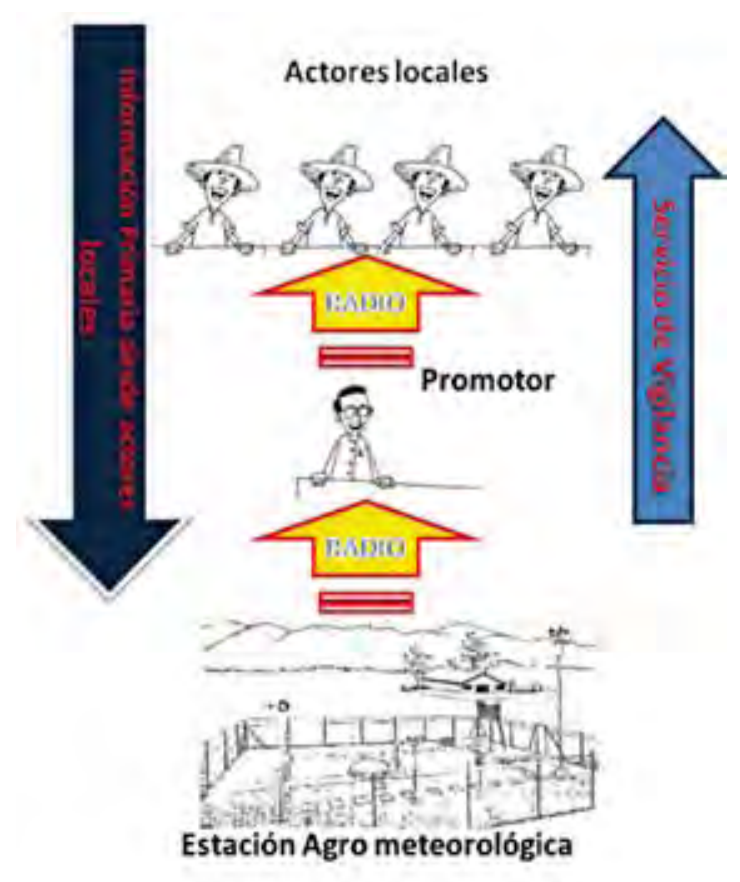

Figura 5: Esquema de integración del Sistema de Alerta Agrometeorológica en el Valle de San Lorenzo.

Fuente: propia.

el valle de San Lorenzo y el proceso de formación permitirán que el agricultor posea criterios técnicos que le facilite su propia construcción del conocimiento y búsqueda de comunicación, participación y expresión [4]. Bajo esta consideración fueron desarrollados:

\section{Talleres formativos}

Estos talleres tuvieron un enfoque agroclimático y de agronegocios (fig. 6). En el enfoque agroclimático se desarrollaron los siguientes temas:

- Problemática ambiental en el valle de San Lorenzo.

- Definición: clima, variables y elementos que la integran.

- Definición de fenología, su importancia: Fases fenológicas de los principales cultivos instalados en el valle San Lorenzo: mango y limón.

- Definición e importancia de la agrometeorología a nivel regional.

Según la figura 5 y la tabla 1, una mayor difusión e intercambio de información agrometeorológica son acciones prioritarias para un sistema de vigilancia agrometeorológica en el ámbito del valle de San Lorenzo.

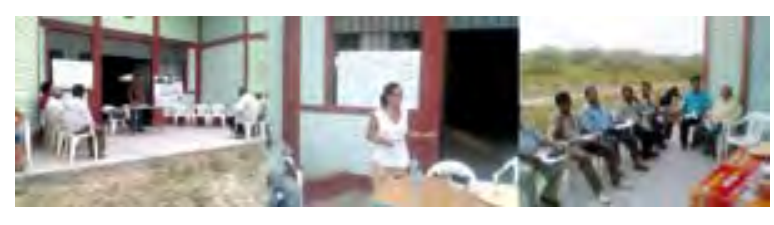

a)

b)

c)

Figura 6: Proceso de formación y capacitación en talleres en el centro comunal del sector La Merced, Valle de San Lorenzo.

Fuente: Dedios, 2010.

Tabla 1: Flujo de información de la Red agrometeorológica en el valle de San Lorenzo.

\begin{tabular}{|l|l|}
\hline \multicolumn{1}{|c|}{$\begin{array}{c}\text { Información de } \\
\text { actores locales }\end{array}$} & \multicolumn{1}{c|}{ Valor agregado } \\
\hline $\begin{array}{l}\text { Observaciones } \\
\text { fenológicas de cultivos. }\end{array}$ & $\begin{array}{l}\text { Predicciones meteorológicas } \\
\text { a corto, mediano y largo } \\
\text { plazo. }\end{array}$ \\
\cline { 2 - 2 } Estado de cultivos. & $\begin{array}{l}\text { Predicciones del balance } \\
\text { hídrico y vigilancia de } \\
\text { la sequía. Monitoreo de } \\
\text { indicadores del balance } \\
\text { hídrico para el cultivo. }\end{array}$ \\
\hline $\begin{array}{l}\text { Deterioro por: } \\
\text { plagas, enfermedades } \\
\text { y eventos } \\
\text { meteorológicos } \\
\text { extremos. }\end{array}$ & $\begin{array}{l}\text { Predicción, vigilancia de } \\
\text { enfermedades y plagas, y } \\
\text { rendimientos. }\end{array}$ \\
\hline $\begin{array}{l}\text { Sobre la aplicación de } \\
\text { labores de manejo del } \\
\text { cultivo. }\end{array}$ & $\begin{array}{l}\text { Recomendaciones de labores } \\
\text { fitotecnias y fitosanitarias. }\end{array}$ \\
\hline $\begin{array}{l}\text { Sobre resultados } \\
\text { productivos. }\end{array}$ & Alertas de eventos extremos. \\
\hline
\end{tabular}

\section{Sistema de difusión}

Un sistema de difusión radial es implementado en el ámbito del valle de San Lorenzo, donde día a día se da a conocer el estado del tiempo y las recomendaciones culturales a seguir en los cultivos instalados, como recomendaciones sobre la frecuencia de riego, podas, fertilización, entre otros. De otro lado, se produce información en diferentes soportes gráficos (afiches, boletines) y audiovisuales (programas didácticos-divulgativos mediante videos, y espacios televisivos).

\section{RESULTADOS Y DISCUSIÓN}

Los resultados indican que aproximadamente 25 actores representantes del valle de San Lorenzo 
conocen el Sistema de Alerta Temprana en el valle de San Lorenzo (Tambogrande, Perú). Con este enfoque, se pretende articular a los actores directos (productores) con aquellos tomadores de decisiones vinculados principalmente con la Junta de Usuarios del valle de San Lorenzo con la finalidad de crear en ellos nuevas capacidades.

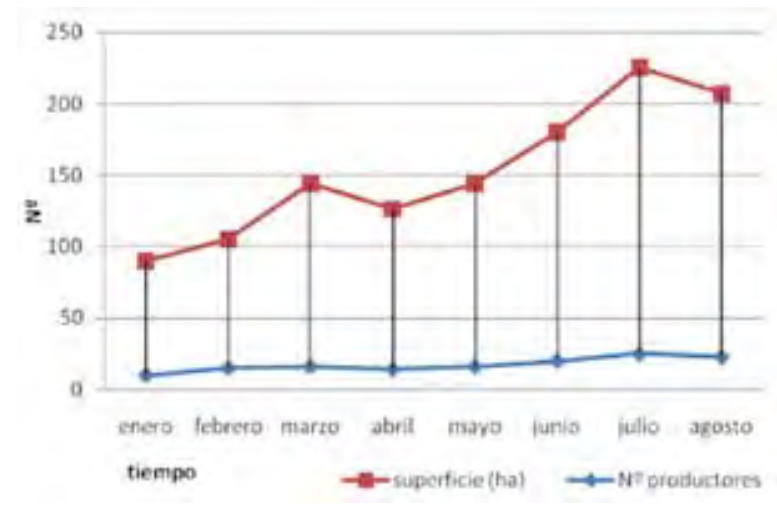

Figura 4: Productores directamente capacitados en el valle de San Lorenzo.

En relación al interés de los agricultores a ser capacitados, observamos que el número va en aumento (fig. 4), si incluimos a aquellos agricultores que toman decisiones a partir de la información recibida por comunicación radial en el ámbito del valle. Esta es una situación importante si consideramos la superficie (ha) de parcelas sobre la cual dichos productores tomarían adecuadas decisiones. De ello observamos, que un promedio de 20 agricultores emplearían la información climática para la adecuada gestión de su parcela.

De otro lado, los productores agropecuarios con un nivel de instrucción básica podrían tener en algunos casos limitaciones para asimilar los nuevos conocimientos y ponerlos en práctica. Por ello, tanto las prácticas agrícolas como las actividades tradicionales desarrolladas por los productores locales condicionan la posibilidad de introducir nuevas propuestas y recomendaciones derivadas de las predicciones. Las experiencias adquiridas desde otras latitudes representan metodologías que constituyen herramientas de enseñanza para fortalecer y prevenir posibles peligros y mitigar daños, además de elevar la capacidad de adaptación a situaciones adversas.

\section{CONCLUSIONES}

Si el clima es un factor determinante para el fracaso o éxito de la producción agrícola, ¿no es lógico empezar a estudiar el clima? A partir de esa reflexión, los campesinos productores de mango y limón del valle de San Lorenzo aprenden a sistematizar su observación y contribuir a reducir su vulnerabilidad ante el cambio climático.

Disponer de una base de información climática fenológica recopilada desde las estaciones agrometeorológicas sobre los principales cultivos y aquellos recientes de gran interés económico no solo en el ámbito del valle de San Lorenzo sino también en la región constituye un fuerte potencial disponible para que los productores tomen decisiones adecuadas.

La escasez de información histórica del valle de San Lorenzo, tanto climática como fenológica, se puede solucionar con el empleo de herramientas que faciliten las posibilidades actuales de automatización, en correspondencia con las concepciones enunciadas por Simeón [5]. Por ello, la capacidad de generar información de observaciones fenológicas y biométricas de cultivos en el radio de acción de las estaciones agrometeorológicas cercanas al valle de San Lorenzo, Partidor y Mallares, representa un sistema de débil correspondencia entre el agricultor y la ciencia. Por esta razón, se evidencia como un problema estratégico general, si se analiza la relación inversa con lo expresado por Núnez [6] al argumentar que la utilización de los resultados precedentes, su modificación permanente y el cruce de información y modelos hace que la ciencia se constituya en una tradición acumulativa de conocimientos y prácticas.

Por ello se ha identificado como uno de los problemas la necesidad de un mayor extensionismo rural. Los resultados de la investigación establecen la necesidad de un sistema de información operativo, desde la edición de boletines y la emisión de avisos tempranos de alerta dirigidos a agricultores y profesionales del sector agrícola en el valle de San Lorenzo, de manera que permita a los agricultores aprovechar de una forma adecuada las condiciones favorables del tiempo y del clima y minimizar las pérdidas cuando estas condiciones sean desfavorables. De 
esta manera, el sistema implementado garantiza la posibilidad de una cobertura informativa para una mejor manera de gestionar y mejorar su producción en los principales cultivos de mango y limón.

La capacitación a los agricultores supone el fortalecimiento de los sistemas observacionales, de gestión y difusión de datos e información meteorológica, biológica y productiva con la integración de actores claves para perfeccionar un sistema de vigilancia, predicción y avisos tempranos de peligros climáticos y agrometeorológicos asociados a la variabilidad y cambio climático, contribuyendo al incremento de la producción sostenible de alimentos, a acciones de adaptación y mitigación en zonas sensibles y a la recuperación de los suelos degradados.

\section{AGRADECIMIENTOS}

Al Concejo Nacional de Ciencias y Tecnologías, CONCYTEC, por financiar el proyecto; al ingeniero Jorge Yerren Suárez del Servicio Nacional de Meteorología y al técnico Félix Zapata del Proyecto Especial Chira-Piura.

\section{REFERENCIAS}

[1] T. J. Crowley y Gerald R. North, «Abrupt Climate Change and Extinction Events in Earth History», Science, (1988).

[2] N. Oreskes, «Beyond the Ivory Tower. The Scientific Consensus on Climate Change», Science 306 (5702): 1686, (2004).

[3] Joung et al., http://ocw.udem.edu.mx/cursos-deprofesional/hu-3110-pensamiento-social-de-laiglesia/M1/6-una-metodologia-de-vida-ver-juzgaractuar/ (2006).

[4] D.H. Jonassen, Computers as mindtools for schools, New Jersey, Prentice Hall, (2000).

[5] R.S. Simeón, "Cuba posee una verdadera riqueza de conocimientos", Ciencia, Innovación y Desarrollo, Revista de Información Científica y Tecnológica, ISSN 1023-1722, Vol. 9, No.2, 2004, 6-8.

[6] J. Núńez, La ciencia y la tecnología como procesos sociales. Lo que la educación científica no debería olvidar, Organización de Estados Iberoamericanos para la Educación, la Ciencia y la Cultura, 2003, en http://www.campus.oei.org/.

E-mail: ndedios@senamhi.gob.pe 